
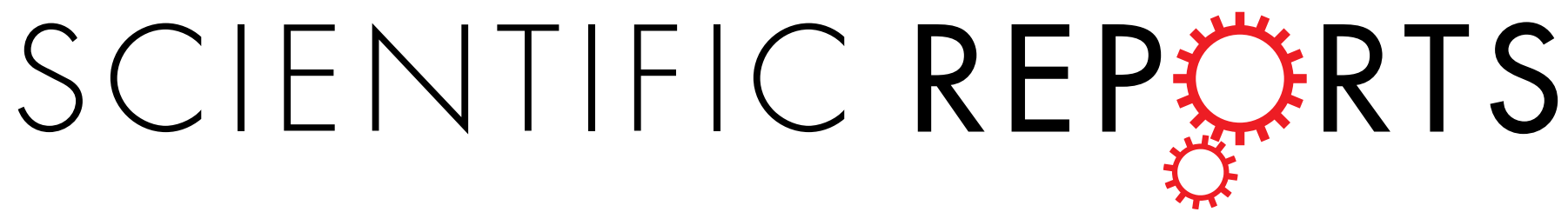

OPEN

Received: 07 March 2016

Accepted: 28 July 2016

Published: 23 August 2016

\section{Charge and spin transport in single and packed ruthenium-terpyridine molecular devices: Insight from first-principles calculations}

\author{
C. Morari ${ }^{1}$, L. Buimaga-larinca ${ }^{1}$, I. Rungger ${ }^{2,3}$, S. Sanvito ${ }^{2}$, S. Melinte ${ }^{4}$ \& G.-M. Rignanese ${ }^{5,6}$ \\ Using first-principles calculations, we study the electronic and transport properties of \\ rutheniumterpyridine molecules sandwiched between two Au(111) electrodes. We analyse both \\ single and packed molecular devices, more amenable to scaling and realistic integration approaches. \\ The devices display all together robust negative differential resistance features at low bias voltages. \\ Remarkably, the electrical control of the spin transport in the studied systems implies a subtle \\ distribution of the magnetisation density within the biased devices and highlights the key role of the \\ Au(111) electrical contacts.
}

The study of metallic complexes has been a mainstay for molecular electronics ${ }^{1,2}$ and metallo-supramolecular chemistry $^{3-5}$. Numerous experimental investigations, accompanied by theoretical approaches ${ }^{6}$, were conducted in the search for possible molecular building blocks for future devices with tunable magnetic behaviour ${ }^{7-12}$. Seminal device fabrication-driven searches have been directed to the study of ordered thin films, from multilayers to monolayer assemblies ${ }^{13-20}$, and addressed the behaviour of single molecules on metallic surfaces ${ }^{21-26}$.

Among the wide variety of candidates for single-molecule two-terminal devices ${ }^{27-29}$, compounds incorporating a single transition metal ion constitute a privileged categor $\mathrm{y}^{30-34}$. In particular, the presence of a transition metal ion allows the tuning of the electronic transport through well-defined charge and possibly spin states of the molecular species. Recent experimental work on charge transport in transition metal coordination complexes revealed a rich physics including inelastic tunneling effects, negative differential resistance and Kondo resonances ${ }^{32,35}$. The case for transition metal coordination complexes is thus twofold. From the experimental point of view, there remains a strong interest to carefully consolidate the salient features that have come off intriguingly from single-molecule electrical measurements, and which will likely be expanded in technologically relevant molecular devices. Equally important, several numerical methodologies developed in the last decade play a considerable part in embodying new physical concepts and in designing critical experiments in molecular electronics. Accordingly, a vivid theoretical effort is currently devoted to the understanding and prediction of the unique charge transport signatures of coordination complexes and metal-organic frameworks ${ }^{36-42}$. Here we study the $\mathrm{Ru}($ tpy - SH) 2 cardan-joint-like molecule, sandwiched between two $\mathrm{Au}(111)$ electrodes, where tpy-SH represents $4^{\prime}-$ mercapto $-2,2^{\prime}: 6^{\prime}, 2^{\prime \prime}-$ terpyridine. The Ru ion is bonded within an approximately octahedral environment to the two terpyridine derivatives linked to the $\mathrm{Au}(111)$ surfaces.

\section{Methods}

The calculations are performed with SIESTA ${ }^{43,44}$ and SMEAGOL ${ }^{45-47}$ codes. We employ Troullier-Martins pseudopotentials $^{48,49}$ and localized basis sets ${ }^{43}$. For the molecules we use a double zeta polarized basis set. For Au we use a single zeta for $5 d$ orbitals and a double zeta polarized basis set for $6 s$ orbitals. On the one hand, our choice is motivated by the computational effort; see below the description of the geometric models used in the calculations. On the other hand, this choice is indeed reasonable since the $5 d$ orbitals of Au lay at about $2 \mathrm{eV}$ below the Fermi

${ }^{1}$ National Institute for Research and Development of Isotopic and MolecularTechnologies (NIRDIMT), 65-103 Donath, Ro-400293, Cluj-Napoca, Romania. ${ }^{2}$ School of Physics and CRANN, Trinity College, Dublin 2, Ireland. ${ }^{3}$ National Physical Laboratory, Teddington, TW11 0LW, United Kingdom. ${ }^{4}$ ICTM Institute, Université catholique de Louvain, 1348 Louvain-la-Neuve, Belgium. 5 IMCN Institute, Université catholique de Louvain, 1348 Louvain-la-Neuve, Belgium. ${ }^{6}$ European Theoretical Spectroscopy Facility (ETSF), 1348 Louvain-la-Neuve, Belgium. Correspondence and requests for materials should be addressed to G.-M.R. (email: gian-marco.rignanese@uclouvain.be) 
(a)

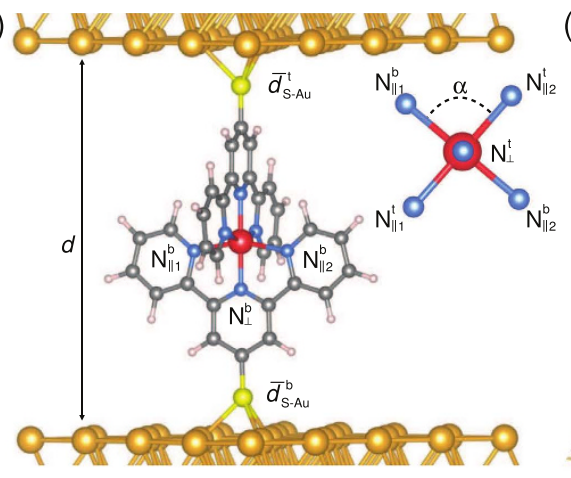

(b)

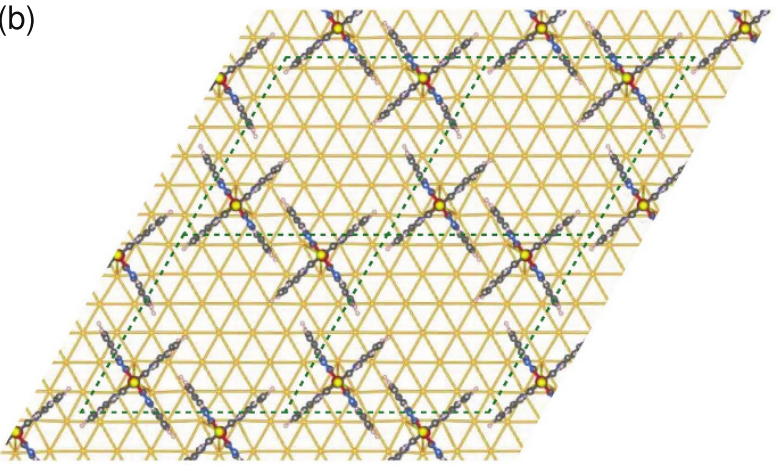

Figure 1. Ball and stick representation of the different models. (a) Side view of single molecule devices (Models 1-6 and 2B without Ru atom) and (b) top view of the packed molecular device (Model 2P). The Au, S, $\mathrm{Ru}, \mathrm{N}, \mathrm{C}$, and $\mathrm{H}$ atoms are in gold, yellow, red, blue, gray, and white. For Model $2 \mathrm{P}$, a $2 \times 2$ unit cell (dotted lines) is represented. For the sake of clarity, only the bottom electrode is shown and it is trimmed to the first atomic layer accommodating the linking $S$ atoms in hollow positions.

level and therefore they do not take part in the transport. We note that alike basis sets were successfully used in previous transport calculations ${ }^{50,51}$. The energy shiff ${ }^{43}$ used to define the Au orbitals is optimized by taking the geometry of the bulk $\mathrm{Au}$ as reference. We found that a $300 \mathrm{meV}$ energy shift for Au leads to a computed bulk lattice parameter of $4.098 \AA$, close to the experimental value of $4.078 \AA$. For the S, Ru, N, C, and H atoms, the energy shifts are chosen employing as reference the geometry of the molecule optimized using a plane-wave method ${ }^{52}$. We inferred that the smallest forces (about $0.4 \mathrm{eV} / \AA$ ) are obtained with a $25 \mathrm{meV}$ energy shift.

For all the investigated geometric models, we use generalized gradient approximation ${ }^{49}$ functionals. We employ periodic boundary conditions in the direction orthogonal to the transport direction, sampling over a uniform $2 \times 2$ grid of $k$-points in the two-dimensional Brillouin zone. In the transport calculations, the Green's function leading to the density matrix is integrated typically over 24 energy points on the complex semi-circle, 16 points along the line parallel to the real axis and 8 poles. The integral over real energies at finite bias is evaluated over about 180 points. The real-space grid cutoff is chosen to give an equivalent plane-wave energy cutoff of 250 Ry.

In all our models, the electrodes consist of five $\mathrm{Au}(111)$ layers. For single molecule devices, we adopt a $5 \times 5$ surface cell resulting in a lattice constant of $20.49 \AA$ based on the optimized lattice constant of bulk Au. When attaching a molecule to the electrodes, the most stable configuration is achieved when the $S$ atom is located at the hollow position with respect to the $\mathrm{Au}(111)$ surface, with a S-Au distance of $\sim 1.8 \AA$ A Therefore, this hollow position is adopted in all models. However, four different distances between electrodes [ $d$ in Fig. 1(a)] are considered, leading to S-Au bond lengths ${ }^{53}$ in line with typical experimental conditions. The corresponding models are referred to as Model 1, Model 2, Model 3 and Model 4, with supercell dimensions in the direction perpendicular to the surface of $37.68,37.98,38.28$, and $38.48 \AA$.

In order to obtain a better sampling of the geometric configurations of the molecule linked to $\mathrm{Au}(111)$ electrodes, different dihedral angles [ $\alpha$ in Fig. 1(a)] for the bridging ruthenium ligand are also considered. The investigation of alike molecular deformations is justified by the fact that experimental investigations are usually carried by inserting the dithiol-tethered ruthenium-bis(terpyridine) complex into an alkanethiol self-assembled monolayer ${ }^{23}$ or via mechanically breakable junction $s^{25}$. We thus take into account two bent conformations with respect to the relaxed structure of the isolated molecule [Fig. 1(a) and Supplementary Information], while keeping the distance between electrodes identical to that used in Model 2. These latter models will be labeled as Models 5 and 6, respectively. Furthermore, in order to better understand the role of the Ru atom in the electronic transport, we also consider Model 2B which is obtained by removing the Ru atom from Model 2. It thus consists of two bare terpyridine units attached to the $\mathrm{Au}(111)$ electrodes and it is specified below as Au-S-tpy - tpy-S-Au.

The mono-molecular devices discussed until now have obvious advantages when considering the fine tuning of the interelectrode distances; however, their performance is seriously restricted to the capability of controlling precisely their molecular geometry. Robust negative differential resistance ${ }^{54,55}$ devices are sought after for technology purposes, and monolayers represent the next step to improve upon the performance of single-molecule devices. Therefore, in order to prove the efficiency of the $\mathrm{Ru}(\mathrm{tpy}-\mathrm{SH})_{2}$ molecules on account of the presence of negative differential resistance behaviour, we studied a more complex two-electrode system (Model 2P) depicted in Fig. 1(b) which mimics the effects arising in self-assembled monolayers. The previously adopted $5 \times 5$ surface cell is too small to accommodate two molecules with a reasonable distance between them. Thus, Model $2 \mathrm{P}$ is built by adopting a $6 \times 6$ surface cell which allows for a minimum distance between the nearest atoms larger than $2.5 \AA$. The distance between the electrodes is chosen to be the same as in Model 2. The two molecules are arranged in a grid-like topology, inducing a porous two-dimensional network.

For all the models, the atomic positions of the molecules are relaxed together with the first two atomic layers of the $\mathrm{Au}(111)$ electrodes until the forces are smaller than $0.01 \mathrm{eV} / \AA$, while keeping the dimension of the supercells constant and pinning the remaining atomic positions of the Au electrodes to their bulk positions. The relaxed 


\begin{tabular}{|l|c|c|c|c|c|c|c|c|c|}
\hline Model & $\mathbf{1}$ & $\mathbf{2}$ & $\mathbf{3}$ & $\mathbf{4}$ & $\mathbf{5}$ & $\mathbf{6}$ & $\mathbf{2}$ & \multicolumn{2}{|c|}{ 2P } \\
\hline$d(\AA)$ & 16.45 & 16.75 & 17.05 & 17.25 & 16.75 & 16.75 & 16.75 & \multicolumn{2}{|c|}{16.75} \\
\hline $\bar{d}_{\mathrm{S}-\mathrm{Au}}^{\mathrm{t}}(\AA)$ & 2.54 & 2.60 & 2.64 & 2.69 & 2.60 & 2.62 & 2.58 & 2.56 & 2.61 \\
\hline $\bar{d}_{\mathrm{S}-\mathrm{Au}}^{\mathrm{b}}(\AA)$ & 2.52 & 2.56 & 2.63 & 2.67 & 2.57 & 2.57 & 2.54 & 2.59 & 2.54 \\
\hline $\bar{d}_{\mathrm{Ru}-\mathrm{N}_{\perp}}(\AA)$ & 2.00 & 2.00 & 2.01 & 2.01 & 2.00 & 2.00 & 1.98 & 2.00 & 2.00 \\
\hline $\bar{d}_{\mathrm{Ru}-\mathrm{N} \|}(\AA)$ & 2.08 & 2.08 & 2.09 & 2.09 & 2.08 & 2.08 & 2.31 & 2.09 & 2.09 \\
\hline$\alpha\left(^{\circ}\right)$ & 88.15 & 88.57 & 88.21 & 88.84 & 89.97 & 89.89 & 89.63 & 86.71 & 86.78 \\
\hline$\Delta E(\mathrm{eV})$ & 0.60 & 0.20 & 0.00 & 0.07 & 0.04 & 0.04 & - & - & - \\
\hline
\end{tabular}

Table 1. Structural properties of the different models: the inter-electrode distance $d$, the average S-Au bond length for the top $\left(\bar{d}_{S-A u}^{\mathrm{t}}\right)$ and bottom $\left(\bar{d}_{S-A u}^{\mathrm{b}}\right)$ electrodes, the average $\mathbf{R u}-\mathrm{N}_{\perp}$ bond length, the average $\mathrm{Ru}-\mathrm{N}_{\|}$bond length, the dihedral angle $\alpha$, and the calculated energy with respect to the most stable model (that is Model 3). In the case of Model 2B (in which there is no Ru atom), the Ru-N distances correspond to half the N-N distances. The two columns for Model $2 \mathrm{P}$ correspond to the two molecules of the unit cell.

structures can be characterized by the bonding of the molecules to the surface, the atomic displacements at the surface, and the molecular geometry deformation as reported in Table 1.

For Models 1 to 6, it is even possible to directly compare the different computed total energies. We found that Model 3 represents the most stable geometry of the molecule in contact with the semi-infinite Au(111) electrodes. Taking Model 3 as a reference, we first note that on pulling the electrodes apart (Model 4), as expected, the energy increases before the breaking of the molecular junction. Second, it can be observed that the reduction of the distance $d$ between the electrodes induces a marked instability: a decrease of the distance between the two gold electrodes by $0.6 \AA$ leads to an increase of the total energy by about $0.6 \mathrm{eV}$. In contrast, the variation of the dihedral angle $\alpha$ for the bridging Ru atom also leads to intriguing behaviour from the energetic point of view: Models 5 and 6 are more stable than Model 2. This is likely a consequence of the fact that the relative bending of the terpyridine units favours a dihedral angle $\alpha$ closer to $90^{\circ}$ and allows thus the S-Au bonds to assume larger length values.

We also found slight differences for the two $S$ atoms linked on the two metallic contacts in terms of the average bond length $\bar{d}_{\mathrm{S}-\mathrm{Au}}$. For each electrode, the differences among the lengths of the S-Au bonds for a given S atom were less than $0.02 \AA$ in all cases, within the numerical accuracy of the method. The smallest differences between $\bar{d}_{\mathrm{S}-\mathrm{Au}}^{\mathrm{t}}$ and $\bar{d}_{\mathrm{S}-\mathrm{Au}}^{\mathrm{b}}$ (where the $\mathrm{t}$ and $\mathrm{b}$ superscripts refer to the top and bottom electrodes, respectively) were observed for the most stable configuration (Model 3), while the largest differences were computed for Models 5 and 6 as well as for the control system provided by Model 2B.

The lengths of the $\mathrm{Ru}-\mathrm{N}$ bonds are also reported in Table 1, distinguishing two values according to their relative orientation ( $\|$ and $\perp$ ) with respect to the surface of the $\mathrm{Au}(111)$ electrodes, as depicted in Fig. 1(a). We observe that the values are in agreement with the previous studies ${ }^{25}$ and are essentially unchanged across the studied mono-molecular devices.

For Model 2P, the inter-electrode distance is $d=16.75 \AA$, similar to Models 2, 5, 6 and 2B. For each of the two molecules of the unit cell, the $\mathrm{Ru}-\mathrm{N}_{\perp}$ bond length is $2.00 \AA$ and the $\mathrm{Ru}-\mathrm{N}_{\|}$bond length is $2.09 \AA$. The dihedral angles are $86.71^{\circ}$ (Molecule 1 ) and $86.78^{\circ}$ (Molecule 2), respectively, for the two Ru(tpy $-\mathrm{S}$ ) accommodated in the unit cell, smaller than those computed for the mono-molecular devices. Due to the packing, the molecules slightly adapt their distances with respect to the electrodes. One of them (Molecule 1) is slightly closer to the top electrode (unlike any of the previous models) with distances $\bar{d}_{\mathrm{S}-\mathrm{Au}}^{\mathrm{t}}=2.56 \AA$ and $\bar{d}_{\mathrm{S}-\mathrm{Au}}^{\mathrm{b}}=2.59 \AA$. In contrast, the other (Molecule 2) is closer to the bottom electrode with $\bar{d}_{\mathrm{S}-\mathrm{Au}}^{\mathrm{t}}=2.61 \AA$ and $\bar{d}_{\mathrm{S}-\mathrm{Au}}^{\mathrm{b}}=2.54 \AA$ very similar to Model 2, 5 and 6. Moreover, this is the largest calculated difference between $\bar{d}_{\mathrm{S}-\mathrm{Au}}^{\mathrm{t}}$ and $\bar{d}_{\mathrm{S}-\mathrm{Au}}^{\mathrm{b}}$.

For the SMEAGOL calculations ${ }^{45-47}$, the extended molecules consist of the geometric models mentioned above (Fig. 1) including only the first three electrode layers. There is no need to use more layers due to the short screening length in Au.

\section{Results}

The computed transmission spectra at zero bias $T(E)$ for all models are presented in Fig. 2 for the energy range from -2 to $2 \mathrm{eV}$ around the Fermi level. For Models 1-6, the most interesting feature is the presence of two sharp peaks for the two spin channels, located near the Fermi level, around $0.1-0.2 \mathrm{eV}$. The existence of these two peaks can be explained as follows. Upon linking of the molecule to the metallic electrodes, the degeneracy of its frontier orbitals (see Supplementary Information) is lifted due to small structural distortions of the terpyridine ligands forming the cardan-joint-like molecule. This splitting is further eased by the proximity of these orbitals to the Fermi level and by the fact that the ligands are positioned differently from the top and bottom electrodes. A comparison with Model 2B, reveals that the presence of the bridging Ru atom has crucial consequences for the calculated transmission and the electrical characteristics of the Au-molecule-Au devices. Several differences between the studied cases can be identified by analysing the $T(E)$ spectra. For instance, referring to the two maxima near the Fermi level, one of the peaks for Models 1-4 has a transmission close to unity, while the other displays a significantly reduced value, in contrast to the allure of the corresponding transmission peaks for Models 5 and 6. We note that for Model 5 the intensities of the spin-resolved transmission channels near the Fermi level are strongly diminished. Qualitatively, a dramatic suppression of the transmission and electrical conductance is indeed expected for a molecule with a dihedral angle $\alpha$ of $90^{\circ}$ between the two rigid terpyridine ligands. 


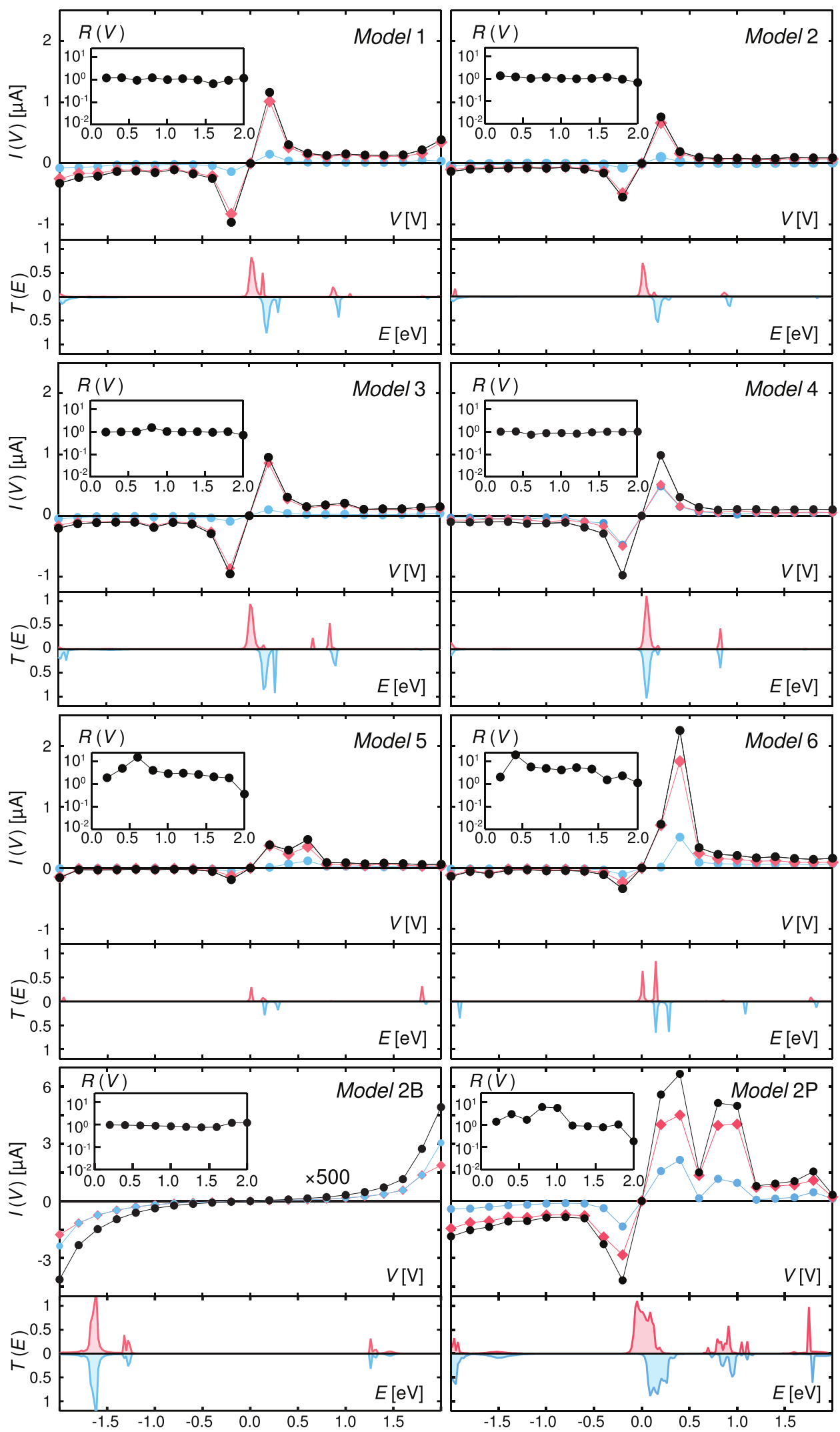

Figure 2. Transport properties of the different models. For all the models, the top panel shows the $I(V)$ characteristics, while the bottom one displays the transmission $T(E)$. The insets report the rectification ratio $R(V)$. Red and blue curves and symbols refer to the spin-up and spin-down components, while black curves and symbols represent the total current. The Fermi level was set to zero. Note that, for the packed molecular model, the reported values have been divided by a factor of 2 for an easier comparison with the results obtained for single-molecule devices. 
While the full width at the half maximum of the transmission peaks located close to the Fermi level also differs significantly among the various studied models and strongly suggests the asymmetry of the interactions of the molecule with the two $\mathrm{Au}(111)$ electrodes, the $T(E)$ data equally displays intriguing peculiarities for Models $1-6$ around $1 \mathrm{eV}$ and in the $E \geq 1.5 \mathrm{eV}$ energy range. For Models $1-4$, relatively strong $T(E)$ peaks at $E \leq 1 \mathrm{eV}$ are accompanying those close to the Fermi energy, in contrast to Models 5 and 6 where the transmission is negligible in the corresponding energy range. In order to investigate the origin of the different $T(E)$ peaks, the $\mathrm{Ru}, \mathrm{S}, \mathrm{C}$ and $\mathrm{N}$ projected density of states (PDOS) was computed for all cases (see Supplementary Information). The most important contributions are those of $\mathrm{Ru}$ and $\mathrm{N}$ atoms for all molecular levels participating in the build-up of the $T(E)$ spectra, including the orbitals adjacent to the Fermi level.

For Model 2P, the calculated transmission spectrum encompasses the contributions of the two molecules, displaying an enhanced mixing of the frontier orbitals and recalling thus the well-known metal-metal coupling mediated by a bridging ligand. The three transmission peaks at about $0.25,0.75$ and $1.75 \mathrm{eV}$ are well spread and display high intensities. Similar to previously discussed molecular models, we attribute these spectral features to hybridised frontier orbitals with enlarged spatial extension, lying above the Fermi level. These broad peaks are in register with marked PDOS features for the Ru and $\mathrm{N}$ atoms (Supplementary Information).

The current-voltage $I(V)$ characteristics for all models are reported in Fig. 2 for biases between -2 to 2 V. For Model 2B, as expected, the transport occurs in the tunnelling regime, with a non-linear $I(V)$ behaviour appearing at high bias without the presence of any resonance phenomena. For the Models 1-6, the $I(V)$ characteristics typically present negative differential resistance (NDR) features with maxima located at $V= \pm 0.2$ and $\pm 1 \mathrm{~V}$, in close agreement with the intensity of the peaks displayed by the $T(E)$ spectra. The investigations which have been carried out so far on the NDR in molecular systems included several classes of local probes and nano-patterned electrodes ${ }^{56-58}$. The NDR has been observed in various molecules accommodating metal atoms ${ }^{59-61}$ and different molecule-electrode interfaces ${ }^{62-65}$. Early attempts to construct devices exhibiting NDR recognised the essential role of the surrounding medium and electrodes ${ }^{61,62,66-69}$. The appearance of nearly symmetric NDR features in molecular junctions can be related to various mechanisms including molecular motion and electron-vibron coupling ${ }^{70}$, as well as complex spatial profiles of the electrostatic potential and variable tunnelling couplings of electrodes to the investigated specimens ${ }^{26}$. In the present case, the molecular charging of the frontier orbitals and their spatial configuration localised on the cardan-joint-like linked terpyridine ligands are playing an essential role for the observed NDR features, in conjunction with the tpy-S-Au orbital overlaps. Indeed, for Models $1-6$, the NDR features originate from the orbital re-hybridization which appears under the applied bias and that dynamically changes the effective coupling between the molecule and the leads ${ }^{71}$. We note that for Models 1-4 nearly symmetric current-voltage curves are computed, and the presence of the second current peak at $V= \pm 1 \mathrm{~V}$ is most discernible for Model 3. Interestingly, the $V=1 \mathrm{~V}$ peak weakens for Model 4, i.e. upon stretching the molecular junction. The use of $\mathrm{Au}(111)$ electrodes with variable inter-distances induces thus structural changes in the bridged molecule as registered by the slightly different shapes of the $I(V)$ curves. By comparing the values of the total current at positive and negative voltage values for Models 1-4, we observe small discrepancies, which are attributed to the non-orthogonality of the two terpyridine units and the differences of the average S-Au bond lengths for the two metallic contacts. The overall asymmetry between the positive and negative biases is enhanced for Models 5 and 6. Indeed, for Models 5 and 6 the $V=0.2 \mathrm{~V}$ peak broadens significantly compared to Model 2 and its maximum seems dictated by the conformation of the molecule. For Model 5 , since the twisted terpyridine units are adopting a perpendicular conformation leading to an almost total electronic decoupling of the two ligands, the shape of the $I(V)$ curve becomes similar to that of Model 2B. In contrast with the present results, when molecules are inserted in self-assembled alkanethiol monolayers ${ }^{23}$, the cardan-joint effect is reduced; thus, this type of devices is mostly prone to redox-reaction-induced switching occurring at higher voltages. For Model $2 \mathrm{P}$, the tuning of the molecular energy levels by biasing the metallic electrodes gives rise to a $I(V)$ characteristic with pronounced wide resonances at the transmission peaks. A non-negligible excess electric current is found to flow for biases corresponding to energies within the $T(E)$ forbidden gap. The monolayer device displays an intriguing multi-peak NDR behaviour: while the packed molecules still display the cardan-joint, symmetric NDR effect at low voltage, our calculations indicate that other mechanisms impact the $I(V)$ curve as well. Indeed, the asymmetric peaks at positive bias voltages 1 and $1.7 \mathrm{~V}$, the latter although of much weaker magnitude, resemble the well-known characteristics of resonant tunnelling diodes ${ }^{54,55}$. Such multi-peak NDR phenomena have been probed in electro-active molecules ${ }^{72}$ and are in agreement with both scenarios involving tunnelling through discrete unoccupied states of the specimens and measurement regimes controlling the redox centres of the molecules ${ }^{23}$.

The bias dependent total transmission as a function of energy is represented in Fig. 3 as contour maps for Model 2, representative for Models 1-6, and Model 2P. Our results for Model 2, at 1 and $2 \mathrm{~V}$, are in line with those reported by Dhungana et al. (ref. 12, Fig. 3, bottom panels, $\sim 1$ and $\sim 2 \mathrm{~V}$ ) for similar devices. In both studies, the transmission peaks are displaced to higher energy values upon increasing the bias voltage. Furthermore, for both our Models 2 and 2P transmission peaks are also present in proximity of the Fermi level within the chemical potential window at low bias voltages, which translates into the appearance of NDR features. We do not expect a dramatic change in the position of these peaks (and hence NDR features) by taking into account more accurate correlation effects (Supplementary Information). Our findings are actually supported by experiments performed at low temperature on flexible alkyl-tailed cardan-joint specimens [ref. 73, Fig. 4 (panel B) and Fig. 5 (panel A)], which show the appearance of NDR features below $1 \mathrm{~V}$ (with stronger intensities as the temperature is decreased). Further details on the relation between the $T(E, V)$ landscape and the appearance of NDR features in the transport characteristics can be found in the Supplementary Information.

The evolution of the spin-resolved and of the total currents is peculiarly interesting for the studied models, considering both the different distances between the $\mathrm{Au}(111)$ electrodes and the connections of the molecule in the two-electrode architectures. The $\mathrm{Au}(111)$ electrodes are non-magnetic. For the Models $2 \mathrm{~B}$ and 4 the spin-up 

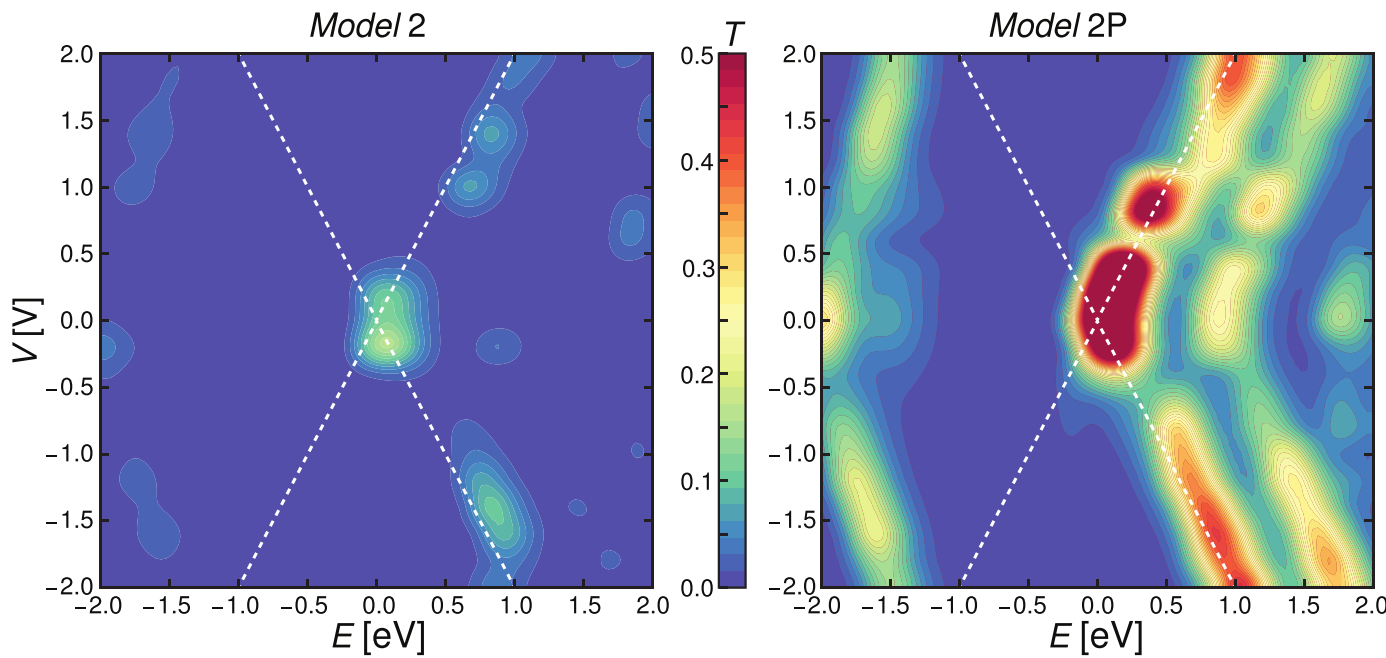

Figure 3. Contour maps of the total transmission $T$ as a function of the energy $E$ and the applied bias voltage $V$ for Models 2 and 2P. The Fermi energy is set to zero on the energy axis. The white dotted lines indicate the chemical potential window as a function of the bias voltage $V$.
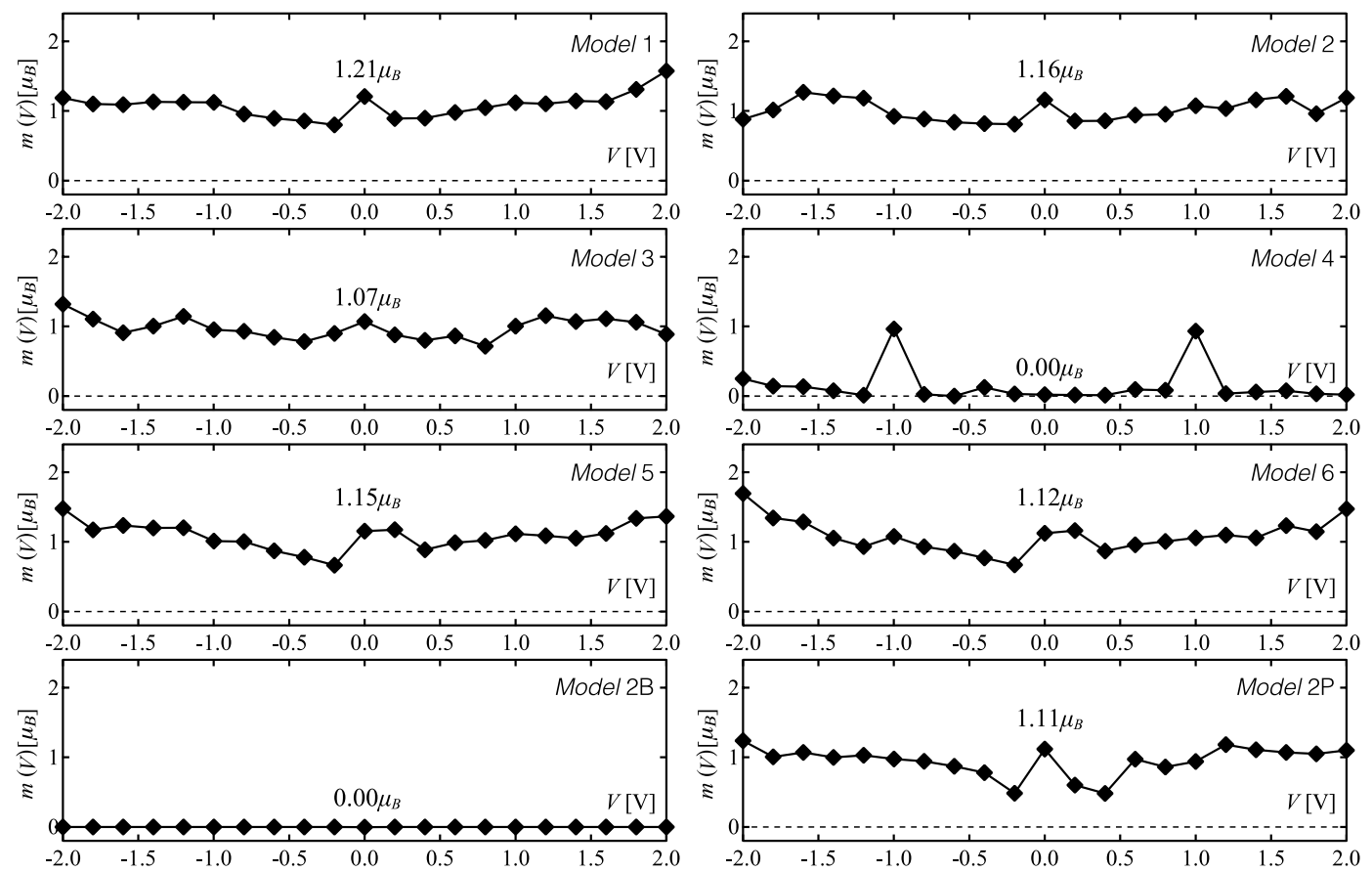

Figure 4. Calculated total magnetic moment $m$ (in $\mu_{B}$ ) as a function of the applied bias $V$ for the Models $1-6,2 B$, and $2 P$.

and spin-down components of the calculated electrical current are almost equal at low biases, suggesting that interfacial and symmetry-breaking, electrode-induced effects are likely not at the origin of the observed negative differential resistance in these devices. Upon shrinking the molecular junction, a highly spin-polarised electric current emerges at low bias for the considered cases: Models 1,2, 3, 5, 6, and 2P. The main contribution to the total electric current arises from the spin-up channel, deriving from the frontier orbitals of the spin-filtering molecule and their preferential coupling with the electronic structure of the electrodes. The consequences on the spin-dependent transport caused by different values of the dihedral angle $\alpha$, can be easily ascertained by comparing the spin polarisation curves $P(V)=\left|I_{\uparrow}(V)-I_{\lrcorner}(V)\right| /\left|I_{\uparrow}(V)+I_{\downarrow}(V)\right|$ for Models 2, 5 and 6 (see Supplementary Information). For Model 2, an effective spin filtration of about $50 \%$ is seen at all biases and for both positive and negative bias voltages. However, for Models 5 and 6, a large spin polarisation of about 100\% is seen at $0.2 \mathrm{~V}$ bias voltage and then it essentially reduces upon increasing the bias voltage. At negative bias voltages, the spin polarisation seems stable around $50 \%$. Thus, the overall spin filtration seems dictated by the electrodes' interdistance, while the dihedral angle and implicitly the average S-Au bond lengths are strongly influencing the 
Model 2
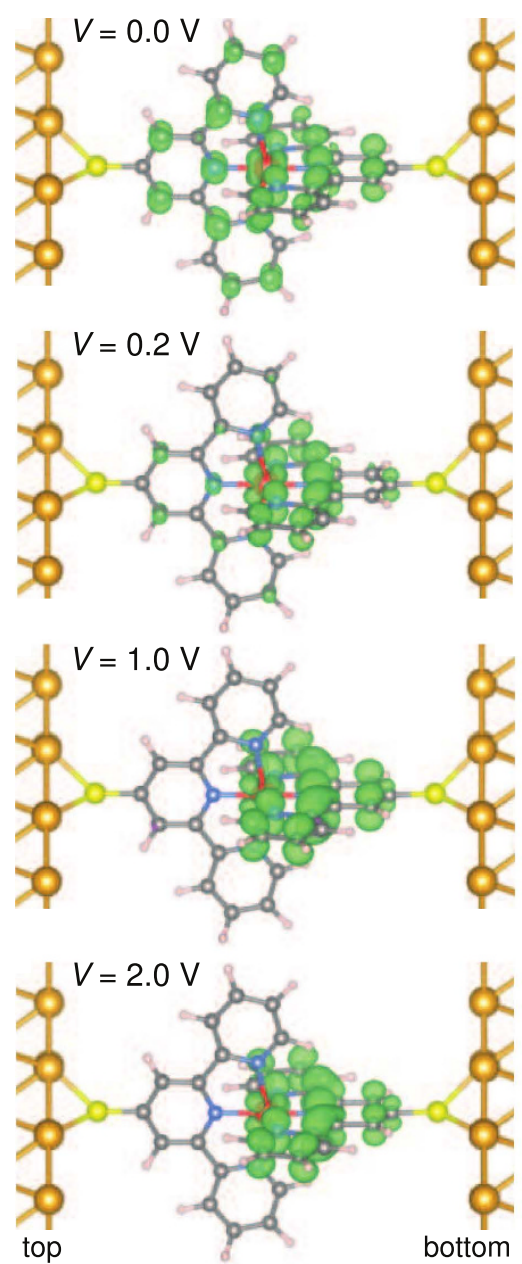

Model 4
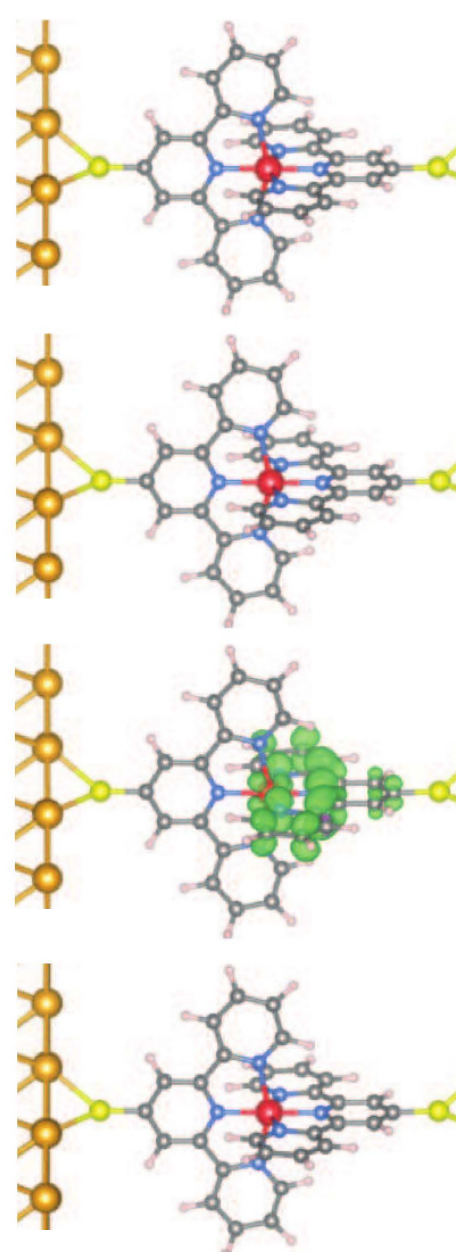
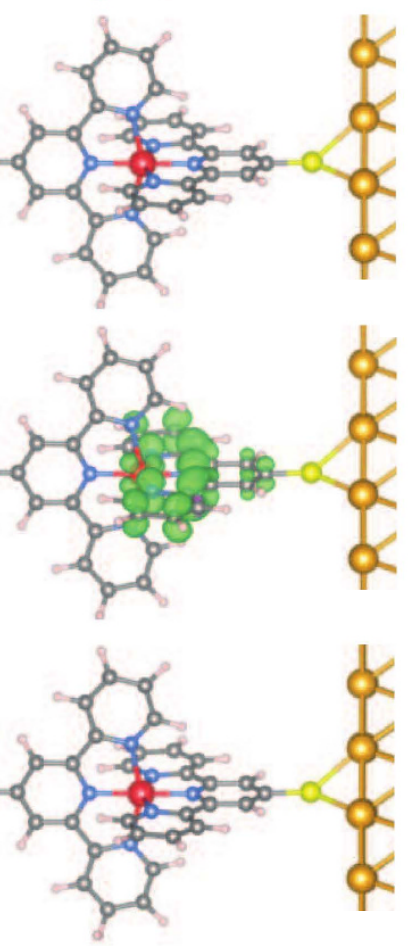
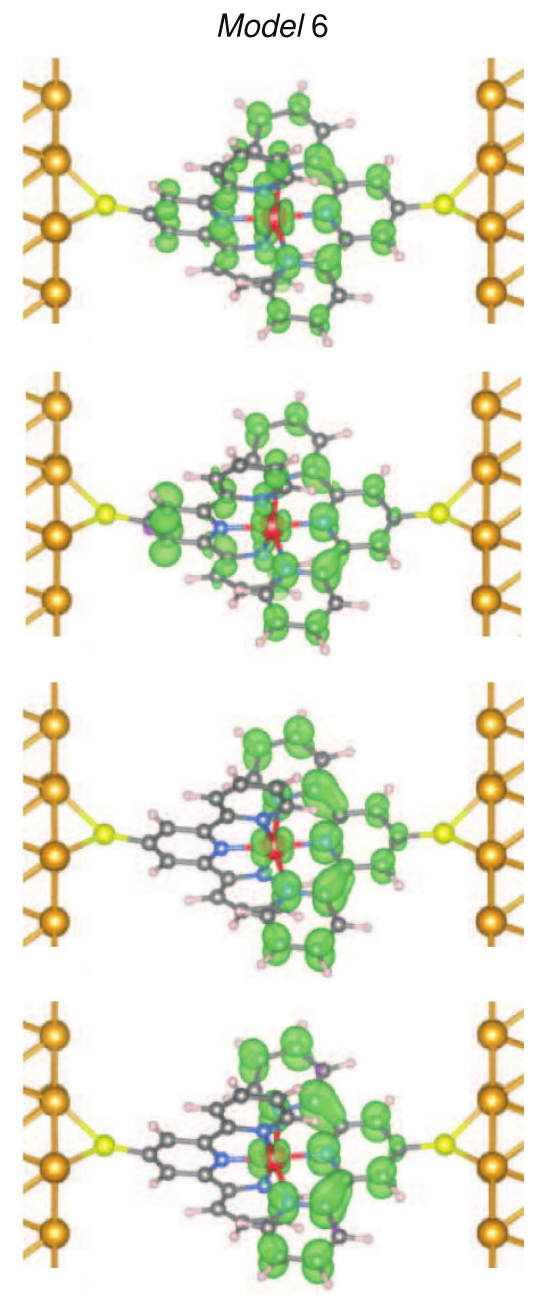

Figure 5. Magnetization density for Models 2,4 and 6 at various voltages $(V=0.0,0.2,1.0$ and $2.0 \mathrm{~V})$. The atoms are represented adopting the same color scheme as in Fig. 1. The isosurfaces in green and violet correspond to a magnetization density of $\pm 2 \times 10^{-3} \mu_{B} /$ Bohr $r^{3}$.

behaviour at low bias voltages. For Model 2P, the overall spin filtration, as evidenced by the $P(V)$ curve (Supplementary Information), is fully consistent to that observed in single-molecule devices.

We now turn to the molecular rectification effects displayed by the different investigated devices (Fig. 2). In agreement with the experimental protocols, we define the rectification ratio $R(V)=|I(V)| /|I(-V)|$ as the ratio of the total current amplitudes for opposite bias polarities. On the one hand, we note that for Models 1-4 rectification values close to unity are computed, since the $I(V)$ curves are only slightly asymmetric and the rectifying character of the molecular devices is thus not enhanced. Obviously, practically no rectification is observed in the control Model 2B involving analogous symmetrical terpyridine ligands. On the other hand, it is quite interesting that for the nanojunction with the largest inter-electrode distance (Model 4), approaching the break junction disposition, unitary values for $R(V)$ are observed at all biases. The rectification $R(V)$ remains thus essentially unchanged when the molecule extends in the gap between the two $\mathrm{Au}(111)$ electrodes, while only upon very slight bending and torsion of the terpyridine ligands, calling for the re-adjustment of the S-Au bond lengths to match a given inter-electrode distance and to adapt towards a $\alpha=90^{\circ}$ conformation (Models 5 and 6), significantly higher values are obtained for $R(V)$ at small biases. The $R(V)$ curve of Model 2P also shows the robust features identified in the study of mono-molecular devices exemplified by the Models 2, 5, and 6 . The monolayers are thus very effective in preserving the rectification ratios of about $\sim 10$ at small applied biases, where the values of the dihedral angle and of the S-Au bond lengths are pivotal. This highlights the ability of the bi-dimensional porous network of $\mathrm{Ru}(\text { tpy }-\mathrm{SH})_{2}$ molecules to respond unequivocally to the applied external bias as exhibited by the $R(V)$ curve.

\section{Discussion}

The calculated values of the total magnetic moment $m$ at different applied biases are summarized in Fig. 4 for all the studied molecular devices [Note that the self-energies of the metallic leads are included in the Hamiltonian]. At zero bias, a total magnetic moment is present for all cases with the exception of Model 2B and Model 4 . 
Precisely, we obtain $m(0)=1.21,1.16,1.07,1.15,1.12$, and $1.11 \mu_{B}$ for Models $1-3,5-6$, and $2 \mathrm{P}$ (for the latter the value has been divided by two in order to take into account the presence of two molecules in the unit cell). It can be seen that $m(0)$ increases when the electrode inter-distance decreases. In contrast, it is much less dependent on the dihedral angle and the packing as can be observed by comparing Models 2, 5, 6, and 2P. When considering the complete $m(V)$ curve, it is less obvious to determine marked trends as a function of the distance, the dihedral angle, or the molecular packing (see superposition of the different curves in the Supplementary Information). However, on the one hand, Model 4 shows a clearly different behavior: the total magnetic moment is very small at all biases but $V= \pm 1 \mathrm{~V}$ for which it shows a peak reaching $m \sim 1 \mu_{B}$. Interestingly, this is the same magnetization value as observed for Models 1-3 at the same bias. On the other hand, for Models 2, 5, 6, and 2P, the dependence on the dihedral angle and implicitly on the S-Au bond length is more pronounced in the low bias regime (for $|V|<0.6 \mathrm{~V})$.

In order to rationalise the differences in the transport and magnetic properties between the different topologies of the molecules connected to the two gold electrodes, the space-resolved spin densities for Models 2, 4 and 6 at various biases are given in Fig. 5. At $V=0 \mathrm{~V}$, for Models 2 and 6 displaying a finite magnetic moment, the magnetic orbital is centered on the metal atom, yet delocalised on both ligands. Interestingly, for $V=0.2 \mathrm{~V}$, the spin density retracts on the terpyridine linked to the bottom electrode for Model 2, while it spreads on both ligands for Model 6. At higher bias voltages, the magnetisation density is located on the bottom terpyridine for both Models 2 and 6 . Note that this is the electrode where $\bar{d}_{\mathrm{S}-\mathrm{Au}}$ attains the smallest value. The same trend as a function of the applied bias was observed for all investigated molecular systems. Apart from the difference observed at low bias voltage, where the molecular conformation plays a major role, Models 5 and 6 behave similarly and in the same way as Models 1, 2 and 3. The magnetisation density plots for Model 2P at various biases are detailed in the Supplementary Information. For Model 4, where a magnetic moment is observed at $|V|=1 \mathrm{~V}$, the space-resolved spin density overlaps the bottom terpyridine. The fact that the magnetic moment is not localised on a single atom (as detailed in the PDOS analysis displayed in the Supplementary Information) precludes the appearance of strong local correlations effects in the transport properties. The different distributions of the magnetisation density observed upon applying a small or a large bias, compared to that calculated at zero bias, could be exploited in the design of networks of molecular devices where the information is encoded by varying the magnetisation density throughout the array ${ }^{73,74}$.

\section{Conclusion}

In summary, we have presented first-principles results for ruthenium-terpyridine molecules sandwiched between two $\mathrm{Au}(111)$ electrodes. Our analysis was performed using several geometrical models for the covalently coupled molecular-metal structures, including devices with single and packed molecules. The spin-resolved transmission spectra and the current-voltage characteristics were calculated for these two-terminal devices. Interestingly, for each investigated model, the presence of $\mathrm{Ru}(\text { tpy }-\mathrm{SH})_{2}$ induces peculiar maxima in the equilibrium transmission function. In particular, their localization very close to the Fermi level leads to distinctive resonance peaks in the current-voltage curves at low bias. The computed electronic properties in the two-terminal devices are spin-dependent and the relative contribution of the two spin channels to the total electrical current and magnetic moment dramatically depends on the applied bias and the interelectrode distance. The studied systems open up new prospects for potential applications in molecular spintronic devices. For instance, the functionalization of the terpyridine units in the $4^{\prime}$ position with donor and acceptor groups, respectively, sitting thus on opposite directions one with respect to the other, could lead to molecular photodiodes with rigid geometries and unique spintronic properties at low temperatures.

\section{References}

1. Aviram, A. \& Ratner, M. A. Molecular rectifiers. Chem. Phys. Lett. 29, 277-283 (1974).

2. Song, H., Reed, M. A. \& Lee, T. Single molecule electronic devices. Adv. Mater. 23, 1583-1608 (2011).

3. Lehn, J.-M. Supramolecular chemistry. Concepts and perspectives (VCH, Weinheim, 1995).

4. Vögtle, F. Supramolecular chemistry. An introduction (Wiley, Chichester, 1993).

5. Sauvage, J.-P. et al. Ruthenium(II) and osmium(II) bis(terpyridine) complexes in covalently-linked multicomponent systems: Synthesis, electrochemical behavior, absorption spectra, and photochemical and photophysical properties. Chem. Rev. 94, 993-1019 (1994).

6. See, for example, for a recent review, Architecture and design of molecule logic gates and atom circuits. Lorente, N. \& Joachim, C. eds. (Springer, Berlin Heidelberg, 2013).

7. Lehmann, J., Gaita-Arino, A., Coronado, E. \& Loss, D. Spin qubits with electrically gated polyoxometalate molecules. Nat. Nanotechnol. 2, 312-317 (2007).

8. Timco, G. A. et al. Engineering the coupling between molecular spin qubits by coordination chemistry. Nat. Nanotechnol. 4, 173-178 (2009).

9. Dediu, V. A., Hueso, L. E., Bergenti, I. \& Taliani, C. Spin routes in organic semiconductors. Nat. Mater. 8, $707-716$ (2009).

10. Vernisse, L. et al. UHV-STM investigations and numerical calculations of a ruthenium $\beta$-diketonato complex and protected ethynyl ligand: $\left[\mathrm{Ru}(\mathrm{dbm})_{2}(\mathrm{acac}-\mathrm{TIPSA})\right]$. J. Phys. Chem. C 116, 13715-13721 (2012).

11. Warner, M. et al. Potential for spin-based information processing in a thin-film molecular semiconductor. Nature 503, 504-508 (2013).

12. Thiele, S. et al. Electrical readout of individual nuclear spin trajectories in a single-molecule magnet spin transistor. Phys. Rev. Lett. 111, 037203 (2013).

13. Liatard, S. et al. An original electrochemical method for assembling multilayers of terpyridine-based metallic complexes on a gold surface. Langmuir 28, 10916-10924 (2012).

14. Tuccitto, N. et al. Highly conductive $\sim 40$-nm-long molecular wires assembled by stepwise incorporation of metal centres. Nat. Mater. 8, 41-46 (2009).

15. Terada, K.-I. et al. Long-range electron transport of ruthenium-centered multilayer films via a stepping-stone mechanism. ACS Nano 6, 1988-1999 (2012).

16. Kurita, T. et al. Surface junction effects on the electron conduction of molecular wires. J. Am. Chem. Soc. 132, 4524-4525 (2010). 
17. Eckermann, A., Feld, D., Shaw, J. \& Meade, T. Electrochemistry of redox-active self-assembled monolayers. Coord. Chem. Rev. 254, 1769-1802 (2010).

18. Gooding, J. J. \& Ciampi, S. The molecular level modification of surfaces: From self-assembled monolayers to complex molecular assemblies. Chem. Soc. Rev. 40, 2704-2718 (2011).

19. Tschan, M. J.-L. et al. Grafting of organoruthenium oligomers on quartz substrates: Synthesis, electrochemistry, optical properties and AFM investigations. Chem. Mater. 19, 3754 (2007).

20. Chen, J., Reed, M. A., Rawlett, A. M. \& Tour, J. M. Large on-off ratio and negative differential resistance in a molecular electronic device. Science 286, 1550 (1999).

21. Wei, Z., Guo, S. \& Kandel, S. A. Observation of single binuclear metal-complex molecules using scanning tunneling microscopy. J. Phys. Chem. B 110, 21846-21849 (2006).

22. Tang, J. et al. Encoding molecular-wire formation within nanoscale sockets. Angew. Chem. Int. Ed. 46, 3892 (2007).

23. Seo, K., Konchenko, A. V., Lee, J., Bang, G. S. \& Lee, H. Molecular conductance switch-on of single ruthenium complex molecules. J. Am. Chem. Soc. 130, 2553-2559 (2008).

24. Hao, X. et al. Direct measurement and modulation of single-molecule coordinative bonding forces in a transition metal complex. Nat. Commun. 4, 2121 (2013).

25. Ruben, M. et al. Charge transport through a cardan-joint molecule. Small 4, 2229 (2008).

26. Xue, Y. et al. Negative differential resistance in the scanning-tunneling spectroscopy of organic molecules. Phys. Rev. B 59, R7852 (1999).

27. Salomon, A. et al. Comparison of electronic transport measurements on organic molecules. Adv. Mater. 22, 1881 (2003).

28. Aradhya, S. V. \& Venkataraman, L. Single-molecule junctions beyond electronic transport. Nat. Nanotechnol. 8, 399 (2013).

29. Mahapatro, A. K., Ying, J., Ren, T. \& Janes, D. B. Electronic transport through ruthenium-based redox-active molecules in metalmolecule-metal nanogap junctions. Nano Lett. 8, 2131-2136 (2008).

30. Park, J. et al. Coulomb blockade and the Kondo effect in single-atom transistors. Nature 417, 722 (2002).

31. Parks, J. J. et al. Mechanical control of spin states in spin-1 molecules and the underscreened Kondo effect. Science 328, 1370 (2010).

32. Ciszek, J. W. et al. Neutral complexes of first row transition metals bearing unbound thiocyanates and their assembly on metallic surfaces. J. Am. Chem. Soc. 128, 3179 (2006).

33. Winter, A., Hoeppener, S., Newkome, G. \& Schubert, U. Terpyridine-functionalized surfaces: Redox-active, switchable, and electroactive nanoarchitectures. Adv. Mater. 23, 3484-3498 (2011).

34. Dhungana, K. M., Mandal, S. \& Pati, R. Switching of conductance in a molecular wire: Role of junction geometry, interfacial distance, and conformational change. J. Phys. Chem. C 116, 17268-17273 (2012).

35. Zhou, J., Samanta, S., Guo, C., Locklin, J. \& Xu, B. Measurements of contact specific low-bias negative differential resistance of single metal organic molecular junctions. Nanoscale 5, 5715-5719 (2013).

36. Datta, S. Electron transport in mesoscopic systems (Cambridge University Press, Cambridge, 1997).

37. Pati, R., McClain, M. \& Bandyopadhyay, A. Origin of negative differential resistance in a strongly coupled single molecule-metal junction device. Phys. Rev. Lett. 100, 246801 (2008).

38. Perrine, T. M. \& Dunietz, B. D. Conductance of a cobalt(II) terpyridine complex based molecular transistor: A computational analysis. J. Phys. Chem. A 112, 2043 (2008)

39. Morari, C. et al. Electronic transport properties of 1,1'-ferrocene dicarboxylic acid linked to $\mathrm{Al}(111)$ electrodes. ACS Nano 3, 4137-4143 (2009).

40. Morari, C., Allmaier, H., Beiuseanu, F., Jurcut, T. \& Chioncel, L. Electronic structure and magnetic properties of metallocene multiple-decker sandwich nanowires. Phys. Rev. B 85, 085413 (2012).

41. Tarafder, K., Kanugo, S., Oppeneer, P. M. \& Saha-Dasgupta, T. Pressure and temperature control of spin-switchable metal-organic coordination polymers from ab initio calculations. Phys. Rev. Lett. 109, 077203 (2012).

42. Tian, Y. et al. Quantum tunneling of magnetization in a metal-organic framework. Phys. Rev. Lett. 112, 017202 (2014).

43. Ordejón, P., Artacho, E. \& Soler, J. M. Self-consistent order- $N$ density-functional calculations for very large systems. Phys. Rev. B 53, R10441-R10444 (1996).

44. Soler, J. M. et al. The SIESTA method for ab initio order- N materials simulation. J. Phys.: Condens. Matter. 14, 2745-2779 (2002).

45. Rocha, A. R. et al. Towards molecular spintronics. Nat. Mater. 4, 335-339 (2005).

46. Rocha, A. R. et al. Spin and molecular electronics in atomically generated orbital landscapes. Phys. Rev. B 73, 085414 (2006).

47. Rungger, I. \& Sanvito, S. Algorithm for the construction of self-energies for electronic transport calculations based on singularity elimination and singular value decomposition. Phys. Rev. B 78, 035407 (2008).

48. Troullier, N. \& Martins, J. L. Efficient pseudopotentials for plane-wave calculations. Phys. Rev. B 46, 1754-1765 (1992).

49. Perdew, J. P., Burke, K. \& Ernzerhof, M. Generalized gradient approximation made simple. Phys. Rev. Lett. 77, 3865-3868 (1996).

50. Stokbro, K., Taylor, J., Brandbyge, M., Mozos, J.-L. \& Ordejón, P. Theoretical study of the nonlinear conductance of di-thiol benzene coupled to $\mathrm{Au}(111)$ surfaces via thiol and thiolate bonds. Comp. Mater. Sci. 27, 151-160 (2003).

51. Toher, C. \& Sanvito, S. Effects of self-interaction corrections on the transport properties of phenyl-based molecular junctions. Phys. Rev. B 77, 155402 (2008).

52. Gonze, X. et al. ABINIT: First-principles approach of materials and nanosystem properties. Comp. Phys. Commun. 180, 2582-2615 (2009).

53. Krüger, D., Fuchs, H., Rousseau, R., Marx, D. \& Parrinello, M. Interaction of short-chain alkane thiols and thiolates with small gold clusters: Adsorption structures and energetics. J. Chem. Phys. 115, 4776-4786 (2001).

54. Esaki, L. New phenomenon in narrow germanium $p-n$ junctions. Phys. Rev. 109, 603 (1958)

55. Chang, L. L., Mendez, E. E. \& Tejedor, C. Resonant tunneling in semiconductors (Plenum, New York, 1991).

56. Andres, R. P. et al. "Coulomb staircase" at room temperature in a self-assembled molecular nanostructure. Science 272, 1323-1325 (1996).

57. Collier, C. P. et al. Electronically configurable molecular-based logic gates. Science 285, 391-394 (1999).

58. Donhauser, Z. J. et al. Conductance switching in single molecules through conformational changes. Science 292, 2303-2307 (2001).

59. Dinglasan, J. A. M., Bailey, M., Park, J. B. \& Dhirani, A.-A. Differential conductance switching of planar tunnel junctions mediated by oxidation/reduction of functionally protected ferrocene. J. Am. Chem. Soc. 126, 6491-6497 (2004).

60. Getty, S. A. et al. Near-perfect conduction through a ferrocene-based molecular wire. Phys. Rev. B 71, 241401(R) (2005).

61. Albrecht, T., Guckian, A., Ulstrup, J. \& Vos, J. G. Transistor-like behaviour of transition metal complexes. Nano Lett. 5, 1451-1455 (2004).

62. Le, J. D., He, Y., Hoye, T. R., Mead, C. C. \& Kiehl, R. A. Negative differential resistance in a bilayer molecular junction. Appl. Phys. Lett. 83, 5518-5520 (2003).

63. Salomon, A., Arad-Yellin, R., Shanzer, A., Karton, A. \& Cahen, D. Stable room-temperature molecular negative differential resistance based on molecule-electrode interface chemistry. J. Am. Chem. Soc. 126, 11648-11657 (2004).

64. Guisinger, N. P., Greene, M. E., Basu, R., Baluch, A. S. \& Hersam, M. C. Room temperature negative differential resistance through individual organic molecules on silicon surfaces. Nano Lett. 4, 55-59 (2004).

65. Rakshit, T., Liang, G.-C., Ghosh, A. W. \& Datta, S. Silicon-based molecular electronics. Nano Lett. 4, 1803-1807 (2004).

66. Wassel, R. A., Credo, G. M., Fuierer, R. R., Feldheim, D. L. \& Gorman, C. B. Attenuating negative differential resistance in an electroactive self-assembled monolayer-based junction. J. Am. Chem. Soc. 126, 295-300 (2004). 
67. Xiao, X., Nagahara, L. A., Rawlett, A. M. \& Tao, N. Electrochemical gate-controlled conductance of single oligo(phenylene ethynylene)s. J. Am. Chem. Soc. 127, 9235-9240 (2005).

68. Cai, L. et al. Reversible bistable switching in nanoscale thiol-substituted oligoaniline molecular junctions. Nano Lett. 5, 2365-2372 (2005).

69. Heersche, H. B. et al. Electron transport through single $\mathrm{M} n_{12}$ molecular magnets. Phys. Rev. Lett. 96, 206801 (2006).

70. Gaudioso, J., Lauzon, L. J. \& Ho, W. Vibrationally mediated negative differential resistance in a single molecule. Phys. Rev. Lett. 85, 1918 (2000).

71. Pemmaraju, C. D., Rungger, I. \& Sanvito, S. Ab initio calculation of the bias-dependent transport properties of $\mathrm{Mn}_{12}$ molecules. Phys. Rev. B 80, 104422 (2009).

72. Mentovich, E. et al. Multi-peak negative-differential-resistance molecular device. Small 4, 55-58 (2008).

73. Seo, K., Konchenko, A. V., Lee, J., Bang, G. S. \& Lee, H. Electron transport processes in on/off states of a single alkyl-tailed metal complex molecular switch. J. Mater Chem. 19, 7617-7624 (2009).

74. Bandyopadhyay, A., Pati, R., Sahu, S., Peper, F. \& Fujita, D. Massively parallel computing on an organic molecular layer. Nat. Phys. 6, 369 (2010).

\title{
Acknowledgements
}

The calculations were performed on the Data Center of NIRDIMT, the Tier-1 supercomputer of the Fédération Wallonie-Bruxelles, and the machines of the Consortium des Equipements de Calcul Intensif en Fédération Wallonie-Bruxelles (CECI). We thank Nicolas Dardenne for performing the hybrid functional and many-body computations and Dr. Xavier Blase for the availability of the FIESTA code. Part of the work (S.M. and G.-M.R) was supported by the Belgian F.R.S.-FNRS in the frame of the FRFC research program (convention no. 2.4510.11) and by the TINTIN (Communauté Française de Belgique, ARC No. 09/14-023) project. S.S. acknowledges financial support from the European Research Council (QUEST project). I.R. acknowledges financial support from the European Union through the FP7 project 618082 ACMOL.

\section{Author Contributions}

C.M. and L.B.-I. carried out the computations with technical support from I.R. and S.S. for the transport calculations. All authors conceived the study, analysed the results, and wrote the paper.

\section{Additional Information \\ Supplementary information accompanies this paper at http://www.nature.com/srep}

Competing financial interests: The authors declare no competing financial interests.

How to cite this article: Morari, C. et al. Charge and spin transport in single and packed ruthenium-terpyridine molecular devices: Insight from first-principles calculations. Sci. Rep. 6, 31856; doi: 10.1038/srep31856 (2016).

\begin{abstract}
(c) (i) This work is licensed under a Creative Commons Attribution 4.0 International License. The images or other third party material in this article are included in the article's Creative Commons license, unless indicated otherwise in the credit line; if the material is not included under the Creative Commons license, users will need to obtain permission from the license holder to reproduce the material. To view a copy of this license, visit http://creativecommons.org/licenses/by/4.0/
\end{abstract}

(C) The Author(s) 2016 